\title{
An Approach to Non-Singular Terms in Discourse
}

Tomek Strzalkowski

School of Computing Science

Simon Fráser University

Burnaby. B.C.. CANADA

V5A 156

\section{Abstract}

A new Theory of Names and Descriptions that offers a uniform treatment for many types of non-singular concepts found in natural language discourse is presented. We introduce a layered model of the language denotational base (the universe) in which every world object is as 5 igned a layer (level) reflecting its relative singularity with respect in other objects in the universe. We define the notion of relative singularity of world objects as an abstraction class of the layermembership relation

\section{Introduction}

Linguistic (and related) literature describes numerous forms of non-singular concepts that can be found in discourse including intensional (or functional) concepts, mass concepts, generic (or general) concepts attributive concepts, abstract concepts, etc. [1]. [2]. [3] [4]. 16] [10] Not all of these approaches could properly capture the distinction between singular and non-singular interpretation of linguistic descriptions, and some were originally devised to deal with singular terms only (such as Donnellan's attributive interpretation of definite descriptions [2]). With the exception of intensional concepts, these notions have not been given satisfactory formal representations that would account for their role in natural language discourse. Perhaps the most successful approach to non-singularity thus far has been presented by Montague [4] with his formalised concept of intension. Unfortunately. the concept of intension does not capture all aspects of non-singularity. and the rigid translation system into intensional logic [4] seems to loose the important aspect of subjectivity in interpreting natural language discourse. Also, the enormous complexity of any non trivial system of possible worlds proved to be a bar in developing a computationally-oriented application of Montague's theory

In this paper we introduce a fragment of a new, and as we believe, computationally feasible Theory of Names and Descriptions that offers a uniform treatment for many types of non-singular concepts found in natural language discourse. Although we limit our presentation to nominal phrase constructions, the approach can be further extended to cover other types of phrases. In our theory we present the formalised definition of non-singularity with respect to a particular discourse situation involving a discourse message, a number of individuals (parties), and their knowledge. beliefs, awareness, etc. We introduce a layered model of reality (the universe) as perceived by a discourse participant. and define the notion of relative singularity of objects in this universe as an abstraction class of the layermembership relation. Subsequently. linguistic descriptions and names are classified as singular. measurably singular, or non-singular depending on what they are assumed to denote in the universe. The relationship between objects addressed in discourse and classified into different layers (levels) of the universe has a particular significance for resolution of certain types of cohesive links in text. We call these links remote references because they cross level boundaries.

\section{Non-singular terms in language}

Many philosophers and logicians, see [1]-[4]. [6]. [10]. appreciate that the usage of the underlined nominal phrases in the following sen tences has a "general" or "generic" character, except for "regular" singular interpretatıons which are possible only in some cases.

\section{Example 1}

(id) The king wears a crown

(1b) The president is elected every four years.

(1c) Gold is a yellow metal

(1d) The temperature is a measure of molecular motion.

One can imagine hundreds of similar examples involving such nonsingular objects as water, heat the Pope, the number etc. Unfortunately. there is no commonly accepted account of these species in philosophical literature Some authors. see [1] and [10]. cautiously called them generic or general (for example the king). or functional (such as the number of students. the temperature) uses of (definite) descriptions. Others. like Kripke [3]. were quite close to consider them names (or at least some of them: heat gold). Yet others. see Quine [6, 7]. advocate the notion of abstract terms as being made of attributes. such as / being] red (further abstracted as redness). or (being/ the man drinking the martini (which cannot be so easily nominalized) which can predicate about "concrete" objects

There are numerous striking linguistic puzzles involving nonsingular definite descriptions, see [1]. [4], [5]. The following example illustrates the phenomenon

Example 2

Consider the following inferences

(2a) The temperature is rising. The temperature is ninety.

so. Ninety is rising

(2b) The president met the Soviet leader many times. The president is Reagan

so. Reagan met the Soviet leader many times.

(2c) The tiger lives in the jung/e. My pet is a tiger. so. My pet lives in the jungle.

The conclusions in (2a) to (2c) are wrong in general case. The explanation given by numerous researchers chiefly amounted to the corroboration that the definite descriptions the temperature, the president and the tiger in the first sentences of (2a). (2b) and (2c) respectively should be interpreted functionally. i.e., as intensions [4] or functions over situations [1]. Observe that if the descriptions were to be interpreted singularly or as enumerating all instances of a nonsingular object (i.e., statements containing them were understood as making claims about each instance) the reasoning would be sound We claim that unless some two descriptions (or names) are used singularly or measurably singularly at the same level no simple reference can be made between them. In fact. another type of reference that we call remote reference can still take place and we shall put this view forward in this paper

\section{The Theory of Names and Descriptions}

Initially let us observe that our language deals with singular objects only. no matter how complex their structure happens to be Suppose somebody is being put into position of the Observer who perceives all these objects and has to use his language to describe them. Some objects are sharply distinguished from others so he chooses to give them names as John. Mary. Fatsy. Sun... The others have no clearly perceivable boundaries but he still may name them: tea. water, grass, snow. ... and then refer to some measurable quantities of them as some tea. little snow. etc. Yet others appear to be numerous, though enumerable. displaying strong similarities to one another. It would be pointless for Observer to give them each a name. Instead he decides to refer to them as a cow. the man. this tree. etc. Still he prefers to say the sun or the lake rather than to invent new names if he is not sure how many of them are there. even if he is aware of just one specimen. Later he may find out that some objects were given identical names. so having encountered them together he must refer to one as the John. the Sun. or a Fatsy. Having completed his job Observer. who is also a part of this world. may name himself Observer or the Observer, and happily sit down under a tree on the grass.

Let us call the whole collection of objects he has just described as the Observer level and use the symbol $L_{0}$ for it. Suppose then we ask Observer to tell us as much as he can about $L_{0}$. Soon he finds 
out that his naming has its limits. As he discovers new facts about his world it becomes more and more cumbersome for him to communicate in terms of every man. some cats, several trees, each president, etc. He discovers that some things he originally considered distinct appear to be instances of some single object. Also he must admit that the identity of some other objects has to be put into question. Being smart enough. Observer invents two new levels, $L_{++1}$ and $L_{-1}$, which augment his world. At level $L_{+1}$ he places the new objects he discovered to be generalisations (or abstractions, if you like) of some measurable amount of objects from $L_{0}$ which displayed a striking similarity or even identity. From the perspective of $L_{+1}$ he is able to tell us that The tiger lives in the jungle, that The president is elected every four years, and that The Morning Star and The Evening Star are actually two appearances of the p/anet Venus. The objects at $L_{+1}$ are singular there but they appear "generic" or "functional" or whatever of that sort as seen from $L_{0}$. Observe that these objects may not have straightforward measurably singular descriptions at $L_{0}$ (like every tiger. some president. etc.). and often it will not be possible to refer to them in the terms of the language available at $L_{00}$. In either case one may expect that some undescribable aspect of an $L_{+1}$ object can emerge at $L_{0}$. even if they all have been derived from $L_{0}$ (which does not have to be the case) Next. Observer invents a new generation of names at $L_{++1}$. the president and the tiger may be among those names. On the other hand. Observer might prefer to use definite descriptions here, for the similar reason lie frequently decided so at $L_{0}$. In fact, we have no means to distinguish between names and definite descriptions in discourse We can only stick to linguistic conventions.

It probably would not take a long time before a new augmenta tion for $L_{++1}$ becomes necessary. Two new levels $L_{+1+1}$ and $L_{++1-1}$ can be added in a much the same fashion. The level $L_{+1-1}$ does not necessarily have to be $L_{0}$. although it probably will. More or less the same happens at the level $L_{-1}$ where Observer can now say that what he previously considered to be the atom actually denotes many different kinds of atoms (H. O Ca. Fe. etc.). that tea is not so uniform and many different teas can be found. and that under the name Joe Smith was actually hidden a group of crime story writers. Subsequently the level $L_{-1}$ will expand by $L_{-1+1}$ and $L_{-1-1}$ with the former often different than $L_{0}$. Let us now formalize our intuition.

Definition 1

A use of a description will be called singular if it denotes or refers to a singular object. A use of a description will be called measurably singular if it denotes or refers to some measurable quantity of a singular object. Otherwise we shall talk of non-singular use.

Definition 2

A level will be an arbitrary collection of singular objects. A level language will contain these and only singular and measurably singular uses of descriptions communicating of the level objects

Definition 3

For any level $L_{n}$, all names appearing in the $L_{n}$ language have singular interpretations.

\section{Definition 4}

For any level $L_{n}$ there will be at least two distinct levels $L_{n-1}$ and $L_{n+1}$ such that $L_{n+1}$ contains the non-singular objects as seen from $L_{m}$ and $L_{n-1}$ contains the objects for which the objects at $L_{n}$ are non-singular

\section{Definition 5}

The Observer level $L_{0}$ is an arbitrary chosen level serving as a refer ence point

Suppose that we have an object $N$ called $N$ at level $L_{0}$. Let $T$ be an arbitrary set we shall refer to as a coordinate. Suppose further that, for the coordinate $T$, the Observer discovers that the identity of $\mathrm{N}$ along that dimension can no longer be accepted. That is. there are at least two $x, y \in T$ such that $N$ at $x \neq N$ at $y$. Without losing generality we can assume that the coordinate $T$ has been chosen so that the following non-equation holds:

- $\forall x, y \in T, x z^{2} y,(N x) \neq(N y)$

Let $(N x)$ denote an object $N_{x}$ for some $x \in T$. The Observer cannot -place $N_{x}^{\prime}$ 's at $l_{-0}$ without violating definitions 2 and 3 Instead he moves them onto a new level $L_{-1}^{N}$ leaving the original object $N$ at $L_{0}$. $N$ may be no longer a "real" object but the concept remains in language. $L_{-1}^{N}$ can be attached to any existing level provided that the definitions 1 to 4 will never be violated. It can also give a beginning to a new level. Note that the distribution of $\mathrm{N}$ over the coordinate $r$ forces other objects from $L_{0}$ to be distributed over $T$ as well, and their instances placed at $L_{-1}^{N T}$ This process may remain mostly implicit until we make an utterance relating $(N x)$ to other objects at $L^{N}{ }^{\top}$. in general we shall say that the level $L_{-1}^{N}$ is lower than the level $L_{0}$. and write $L_{-1}^{N}<L_{0}$ Often we shall drop the superscripts $N$ and $r$ over the level symbol assuming some lower level $L_{-1}$ whenever it does not lead to ambiguity Observe that with the above account the level structure of objects has a dynamic, ever-changing character. Ary new empirical fact to be added to our world knowledge bears a potential reveberation in the level structure involving creation of new levels and moving objects between levels. At probably noft-frequent idle states the definitions 1 to 4 assure the structure balance.

Moving at level $L_{-1}$ the Observer is aware of an enumerable collection of different objects $N_{x}$ 's Extending the description used for $N$ over $N_{x}$ 's the Observer refers to them as the $N$, a $N$. some $N(s)$. every $N$. etc. It is possible, of course, that some other object $N$. found at $L_{0}$ is now disclosed to be an $N_{x}$ for some $x \in T$. What that means is that we have wrongly placed $N^{\prime}$ at $L_{0}$. because it actually belonged to $L_{-1}$. But this was right at the time $N^{\prime}$ was placed there, i.e. it mirrored the state of our knowledge of the world at the time. We may now give names to some $\mathbf{N}_{x}$ 's and $\mathbf{N}$ can very well happen among them. This time however $N$ will not denote the old object from $L_{0}$; this will be actually quite a different name referring to one selected $N_{x}$, and which may be replaced by a definite description of $(N x)$

On the other hand. suppose we have some objects $N_{1}, N_{2}$. considered distinct at $L_{0}$. Suppose then that we discover some resemblance between them along some dimension (coordinate) $T$. so that we need a generalizing concept to talk about them. We climb to some higher level $L_{+1}^{N T}$. i.e. $L_{0}<L_{+\cdot 1}^{N T}$, and establish a new object a superobject. N there. Now as seen from $L_{+1}^{N}$ all $N_{i}$ s are just the occurences of $N$ at $L_{0}$ at different values of coordinate $T$. In other words, the following equation holds:

- $\forall i-i x, x \in T .(N x)=N_{i}$

Observe also that all $N_{i}$ 's now belong to the level $L_{+1-1}^{N}$ which is a part of $L_{0}$. As before we shall drop superscripts $N$ and $T$ for simplicity. No matter how we name $N$ at $L_{+1}$ the following Formula of Discovery summarizes our action:

(FD) $\forall x \forall y, x, y \in T .(N x)=(N y)$

Remember that the formula $F D$ is valid only when observed from $L_{+1}$ At $L_{0}$. $\mathbf{N}_{i}$ 's remain distinct. traditionally - so they remain distinct in the language as well. The generalisation of other objects from $L_{0}$ onto $L_{+1}^{N} T$ may follow but, as in the case of decomposition discussed above. the process will remain largely implicit. Once the superobject $N$ has been created it begins to live its own life. Some new objects from $L_{0}$. different than $N$; s, may now become instances of $N$ at some not yet utilized values of coordinate $T$. Also, we may use descriptions $(N x)$ without caring whether they actually refer to any objects at $L_{0}$. The latter property of general terms which is widely discussed by Quine [6. 7] gets a formal explanation in our theory. It is important not to confuse a superobject with a set $\mathrm{S}$ of lower level instances over some coordinate $T$ as we would obtain a measurably singular concept only. Instead. a superobject can be identified with the function $N$ from $T$ into $L_{0}$ such that whenever $s \in S$ then there is a $t \in T$ such that $(N t)=s$, and then extended arbitrarily beyond the set $S$

Example 3

We have the following distinct object at some level $L_{0}: V$ called Venus. MS called Morning Star, and ES called Evening Star. Upon discovery that they all are just occurences of the same planct we create a new object $V^{\prime}$ named Venus at some level $L_{+1}^{V_{1}^{\prime}} \cdot T$ and such that for some $x, y, z \in T$, where $T$ is a time coordinate, $\left(V^{\prime} x\right)=V\left(V^{\prime} y\right)=M S .\left(V^{\prime} z\right)=E S$. According to the FD formula we conclude from $L_{+1}$ that $V=M S=E S$, while the same conclusion made at $L_{0}$ is false

Example 4

At level $L_{0}$ the Observer is aware of the object TP named The President. Let $T$ be the time coordinate (different than in the last 
example). At $L_{0}$ we have according to the FD formula that

- $\forall x \forall y, x, y \in T(T P x)=(T P y)$

Later the Observer may dicover that for some $t_{1}, t_{2} \in T .\left(T P t_{1}\right)=N$ and $\left(T P t_{2}\right)=R$, and that at some level $L_{-}^{T} P . r$ where $N$ and $R$ belong they are considered distinct and named Nixon and Reagan respectively. But at $L_{0}, R=N$ is true. The last observation can be made clearer if one imagines that TP is some abstract individual which (like Venus) when observed in early $70^{\prime} \mathrm{s}$ is named Nixon. while when observed in 80 's is called Reagan. $\square$

Definition 6

An object $N$ at a level $L_{n}$ is said to be remotely referenced if the reference comes from some level $L_{m}$ such that either $L_{n}<L_{m}$ or $L_{m}<L_{n}$

Typical cases of remote references in discourse have been listed in Example 2 .

\section{Superobjects}

Let us now examine the nature of superobjects i.e.. the objects placed at level $L_{+1}$. It turns out that the plural terms. e.g. presidents, tigers, etc., are actualiy prototypes of superobjects, see $[6]$. and they should therefore be placed at the same level as respective superobjects. We will see that the generalization leads naturally to plural terms which may or may not induce equivalent singular superobjects. Conversely, a plural equivalent to a superobject may suggest the most natural coordinate to decompose the latter onto some lower level. When a superobject lacks a plural equivalent however. we may admit that this object's origin has been traced down. A further decomposition is still possible but this process may often produce objects that will never assume an independent status and will remain recognized only as instances of this general concept scattered over that or another coordinate. This phenomenon is characteristic of the so-called mass objects and their corresponding mass terms. Quite naturally the question of where one level ends and another begins arises The following example gives some insight into the problem of level boundaries.

Example 5

Consider the following sentences

(5a) Mary brings (some) water every day

Let water in ( $5 a$ ) be the name of some superobject $w$ at the level $L_{+1}$. Presumably Mary brings only a part of $w$ but we can say that $w$ is being brought by Mary every day. This is the same $w$ every day. although each time possibly a different part of it is in transit, which leads to the obvious translation (at $L_{+1}$ )

(i) $5 a \rightarrow(b r-e-d M w)$

where $b r-e-d$ stands for brings every day.

On the other hand. suppose that Mary brings some water every day Except for the above interpretation. we also have the measurably singular reading at $L_{0}$ where $W$ is scattered over some coordinate $T$ so that $\exists t \in T$ such that $(w t)$ is being brought by Mary, i.e..

( $\mathrm{t}(\operatorname{br} M(w t)))$. This clause is, of course, relative to every day so at $L_{0}$ we could have

(ii) $5 a \rightarrow(\forall x(d x) \supset(-1 t(b r M(w t))))+$

where brings $\rightarrow$ br. day $\rightarrow d$

Both translations are essentially equivalent, and this equivalence is by no means accidental. It lends a strong support for our Theory of Names and Descriptions. and explains the intuition underlying its formulation.

\section{Conclusion}

In this paper we presented a new approach to representing various kinds of non-singular concepts in natural language as the Theory of Names and Descriptions. The major observation of the Theory is that reality. as perceived by an intelligent individual. can be regarded as a partially ordered structure of levels such that each level contains only those objects which are considered relatively singular Observe

+ To be precise we should represent Mary as $(\mathrm{M})$ here. i.e. as an instance of the $L_{+1}$ object $M$ at some $1 \in T$. However, our naming convention discussed in section 3 allows for replacing the definite description by a new name at the level $L_{0}$. We shall utilize this option here that there are virtually no restrictions imposed upon the notion of relative singularity so that the distribution of objects between levels of the world model may differ among different individuals. Nonsingular objects. called superobjects, are placed at a number of higher levels which are related to the current level with various coordinates. Conversely. a singular object may be decomposed along a coordinate. and new objects. so obtained, will be placed at some lower level. This same coordinate can be used then to obtain instances of other objects at this lower level. so that the relative singularity of objects within each level is maintained. For more details concerning various aspects of the theory the reader is referred to [8] and [9]

\section{Acknowledgements}

The author would like to thank Dr. Nick Cercone and Dr. Jim Delgrande for their comments and suggestions that helped to improve the quality of this paper This research was supported in part by the Natural Science and Engineering Research Council of Canada under Operating Grant number A4309, by the Office of the Academic VicePresident. Simon Fraser University, and by the SFU's Open Graduate Scholarship Thank the LCCR for use of facilities

References

[1] Barwise. J J Perry (1983). Situalions and Attitudes. The MIT Press

[2] Donnellan. K (1971) "Reference and Definite Descriptions." In D. D. Steinberg. L. A. Jakobovits (eds.), Semantics. Cambridge University Press, 100-114.

[3] Kripke. S. (1972) "Naming and Necessity." In D. Davison. G Harman (eds.). Semantics of Natural Language. Reidel. Dordrecht. 253-355

[4] Montague. R. (1974). Formal Philosophy, (R. Thomason, ed.) Yale University Press

[5] Partee, B. H. (1972) "Opacity, Coreference, and Pronouns." In D. Davison. G. Harman (eds.). Semantics of Natural Language Reidel. Dordrecht. 415-441

[6] Quine W V (1960). Word and Object The MIT Press. Cambridge. Mass

[7] Quine. W. V. (1973). The Roots of Reference. Open Court. La Salle. Illinois.

[8] Strzalkowski. T. (forthcoming). A Theory of Stratified Meaning Representation Doctoral Dissertation. School of Computing Science. Simon Fraser University. Burnaby, B.C.

[9] Strzalkowski. T., N Cercone (1985). "A Framework for Comput ing Extra-Sentential References." Proceedings of TANLU Workshop. Halifax. Nova Scotia. 107.116

[10] Vendier. Z. (1971). "Singular Terms." In D. D. Steinberg. L. A. Jakobovits (eds.), Semantics. Cambridge University Press. 115-133. 